\title{
Membrane Tension in Swelling and Shrinking Molluscan Neurons
}

\author{
Jianwu Dai, ${ }^{1}$ Michael P. Sheetz, ${ }^{1}$ Xiaodong Wan, ${ }^{2}$ and Catherine E. Morris ${ }^{2}$ \\ ${ }^{1}$ Department of Cell Biology, Duke University Medical Center, Durham, North Carolina 27710, and ${ }^{2}$ Neurosciences, Loeb \\ Institute, Ottawa, Ontario, Canada, K1Y 4E9
}

\begin{abstract}
When neurons undergo dramatic shape and volume changes, how is surface area adjusted appropriately? The membrane tension hypothesis-namely that high tensions favor recruitment of membrane to the surface whereas low tensions favor retrieval-provides a simple conceptual framework for surface area homeostasis.

With membrane tension and area in a feedback loop, tension extremes may be averted even during excessive mechanical load variations. We tested this by measuring apparent membrane tension of swelling and shrinking Lymnaea neurons. With hypotonic medium (50\%), tension that was calculated from membrane tether forces increased from 0.04 to as much as 0.4 $\mathrm{mN} / \mathrm{m}$, although at steady state, swollen-cell tension $(0.12$ $\mathrm{mN} / \mathrm{m}$ ) exceeded controls only threefold. On reshrinking in isotonic medium, tension reduced to $0.02 \mathrm{mN} / \mathrm{m}$, and at the substratum, membrane invaginated, creating transient vacuolelike dilations. Swelling increased membrane tension with or without BAPTA chelating cytoplasmic $\mathrm{Ca}^{2+}$, but with BAPTA,
\end{abstract}

Biological membranes expand elastically by $<3 \%$ before rupture (Nichol and Hutter, 1996). Beyond this limit, membrane area must be augmented to avoid lysis. Membrane capacitance changes suggest that during swelling, membrane is added from internal stores and that on shrinking, excess membrane is reinternalized (Sukhorukov et al., 1993; Wan et al., 1995). In neurons and muscle cells (Krolenko et al., 1995; Reuzeau et al., 1995), membrane invaginates as vacuole-like dilations (VLDs) during the shrinking phase of swell-shrink cycles, effects that are mechanically mediated.

Although mechanical regulation is unequivocally of physiological significance for various cytoplasmic mechanoenzymes (Pybus and Tregear, 1975; Smith et al., 1995), the meaning of membrane tension effects on ion channel gating remains uncertain (Sachs and Morris, 1998). Membrane tension as a mediator in the disposition of membrane in animal cells, including neurons, is an even newer area of inquiry (Fink and Cooper, 1996; Sheetz and Dai, 1996; Morris et al., 1997).

For neurons and other cells, it has not yet been tested but is assumed that membrane tension increases and decreases in swelling and shrinking, respectively. Most assessments of membrane tension have relied on surface deformations (Waugh et al., 1992;

\footnotetext{
Received March 27, 1998; revised June 9, 1998; accepted June 16, 1998.

This work was supported by a grant from National Institutes of Health to M.P.S. and grants from Natural Sciences and Engineering Research Council, Canada and the Heart and Stroke Foundation of Ontario (T3461) to C.E.M. We thank Dr. G. Banker's lab for providing hippocampal neuron cells.

Correspondence should be addressed to Dr. Catherine E. Morris, Neurosciences, Loeb Institute, Ottawa Hospital, 725 Parkdale Avenue, Ottawa, Ontario, Canada, K1Y 4E9.

Copyright (C) 1998 Society for Neuroscience $\quad 0270-6474 / 98 / 186681-12 \$ 05.00 / 0$
}

unmeasurably large (although not lytic) tension surges occurred in approximately two-thirds of neurons. Furthermore, in unarborized neurons voltage-clamped by perforated-patch in $50 \%$ medium, membrane capacitance increased $8 \%$, which is indicative of increasing membrane area.

The relatively damped swelling-tension responses of $\mathrm{Lym}$ naea neurons (no BAPTA) were consistent with feedback regulation. BAPTA did not alter resting membrane tension, but the large surges during swelling of BAPTA-loaded neurons demonstrated that $50 \%$ medium was inherently treacherous and that tension regulation was impaired by subnormal cytoplasmic $\left[\mathrm{Ca}^{2+}\right]$. However, neurons did survive tension surges in the absence of $\mathrm{Ca}^{2+}$ signaling. The mechanism to avoid hightension rupture may be the direct tension-driven recruitment of membrane stores.

Key words: surface area; mechanosensitive; cell volume; BAPTA; laser tweezers; vacuole-like dilations
Evans and Yeung, 1994). Laser tweezers provide a refined approach for adherent cells: a membrane tether (Fig. 1) is formed, and the force required to keep it at constant length is measured (Dai and Sheetz, 1995a-c). In-plane tension, membrane bending stiffness, and membrane-cytoskeleton interactions all contribute to this static tether force. Separating these contributions in complex cells presents a major difficulty, so they are combined in an apparent membrane tension (Dai and Sheetz, 1995c; Hochmuth et al., 1996; Sheetz and Dai, 1996).

Here we use membrane tethers to estimate tension in osmotically perturbed molluscan neurons. As these neurons swell and shrink, capacitance changes $\left(\sim 0.7 \mu \mathrm{F} / \mathrm{cm}^{2}\right)$ roughly account for changing cell size (Wan et al., 1995). With shrinkage, membrane invaginates at the substratum, forming large $(\sim 1-10 \mu \mathrm{m})$ VLDs (Reuzeau et al., 1995); reswelling reverses them (Fig. 2). VLDs echo the membrane invaginations of regulatory volume decrease (Czekay et al., 1994) and the reversible vesicles of plant protoplasts undergoing osmocytosis (Wartenberg et al., 1992) and of shrinking muscle (Krolenko et al., 1995). VLD formation and reversal are $\mathrm{Ca}^{2+}$-insensitive (Herring et al., 1998).

A working hypothesis is that VLDs represent the initial retrieval phase for the excess membrane realized by osmotic swelling. For minor perturbations, membrane area adjustments go unnoticed, but abrupt swell-shrink perturbations engage large quantities of membrane and result in easily detected VLDs. In vivo, neurons seldom experience such insults, but surface area regulation would be required after secretion (Fujimoto and Ogawa, 1989) and growth-related changes. Neurite tension regulates outgrowth, and anisosmotic media can mimic effects of 
Figure 1. Measuring membrane tension by pulling tethers. Membrane tension is calculated from the static tether force. Note that two distinct features of the plasma membrane are thought to work together to yield the total membrane tension: the bilayer in-plane tension and interactions between membrane and cytoskeleton. Although tethers lack cytoskeleton, they act as tension probes for the cellular plasma membrane where cytoskeleton-bilayer interactions are a factor. Such interactions will differ at free and adherent (dorsal and substratum) surfaces. The region of membrane whose tension is monitored in these experiments is the dorsal surface from which tethers are pulled. By contrast, while monitoring membrane capacitance electrophysiologically or membrane disposition visually, we considered both free and adherent membranes, an important point when relating membrane tension to other variables. The status of cortical actin in osmotically swollen cells is disputed (swelling-induced F-actin disorganization may represent depolymerization and/or disorganization of filaments). Reduced bilayer-F-actin interactions would lower membrane tension estimates but the ability of swollen Lymnaea neurons to actively writhe (Wan et al., 1995) suggests that they retain cortical F-actin (also see Lin et al., 1995).

tension (Bray et al., 1991; Heidemann and Buxbaum, 1994; Lin et al., 1995).

Neuronal membrane tension is $\sim 10^{-2} \mathrm{mN} / \mathrm{m}$ (Hochmuth et al., 1996). Might tension vary sufficiently during swell-shrink excursions to impact directly on channels, or is tension regulated near some low set-point? Answers to such fundamental questions require a quantitative knowledge of tension in osmotically perturbed cells and are important for the intimately related issues of cell surface area regulation, cell volume regulation, and cell shape regulation.

\section{MATERIALS AND METHODS}

Lymnaea stagnalis neurons were prepared as described previously (Reuzeau et al., 1995) but were grown on uncoated $60 \times 22 \mathrm{~mm}$ No. 1 coverslips in medium-filled wells whose walls were a $\sim 40 \times 20 \mathrm{~mm}$ line of silicone grease. The cells were maintained in a damp chamber for 1-4 $\mathrm{d}$ before use. Just before an experiment, a double-coverslip chamber was prepared by mounting the $60 \times 22 \mathrm{~mm}$ coverslip with grease on a cut-away aluminum slide and then lowering a $22 \times 22 \mathrm{~mm}$ No. 0 coverslip onto the silicone grease walls, leaving a $\sim 100-150 \mu \mathrm{m}$ medium-filled gap between small and large coverslip. Excess silicone grease and medium were removed, and the intracoverslip gap was secured with dabs of low melting-point wax at the corners. The assembly was mounted on the microscope stage's slide holder. Rapid solution exchanges were effected (or bead suspensions were added) using a wick system as follows: a drop of the exchange solution was placed at the end of one open edge of the coverslip chamber, and tapered strips of filter paper were placed in contact with the medium at the other open edge. All procedures on Lymnaea neurons were performed at room temperature $\left(23-25^{\circ} \mathrm{C}\right)$. Rat hippocampal neuron cultures were prepared as described previously (Goslin and Banker, 1992) and were maintained at $\sim 37^{\circ} \mathrm{C}$ on the microscope stage using a chamber described previously (Dai and Sheetz, 1995a).

For latex bead preparations, rat IgG (Sigma, St. Louis, MO) was prepared at a concentration of $1.0 \mathrm{mg} / \mathrm{ml}$ in $50 \mu \mathrm{l}$ of Dulbecco's PBS (Life Technologies, Grand Island, NY). Twenty-five microliters of 0.5 $\mu \mathrm{M}$ Covaspheres MX reagent (Duke Scientific, Palo Alto, CA) were added to the $\operatorname{IgG}$ solution and allowed to incubate for $\sim 20 \mathrm{hr}$ at $4^{\circ} \mathrm{C}$. After incubation with $\operatorname{IgG}$, the beads were then washed three times with $0.5 \%$ BSA in PBS by centrifugation at $10,000 \mathrm{rpm}$ and $4^{\circ} \mathrm{C}$ for $10 \mathrm{~min}$ in a microcentrif uge (Beckman Instruments, Fullerton, CA). The final bead pellet was resuspended in $75 \mu \mathrm{l}$ of $0.5 \%$ BSA. The bead solution was diluted 3:100 in culture medium and then sonicated before being added to the cells. With the flow cell mounted on the microscope, the IgGcovered latex beads and then the treatment solutions such as hypotonic medium were exchanged for the normal medium. The samples were viewed by a video-enhanced differential interference contrast (DIC) microscope (IM-35 microscope; Zeiss, Oberkochen, Germany) fitted with oil immersion condenser and objectives and with a fiber optic illuminator. The laser trap consisted of a polarized beam from an $11 \mathrm{~W}$ TEM00-mode near-infrared (1064 nm) laser (model C-95; CVI Corporation, Albuquerque, NM) that was expanded by a $3 \times$ beam expander (CVI Corporation) and then focused through an $80 \mu \mathrm{m}$ focal length achromatic lens (Melles Griot, Irvine, CA) into the epifluorescence port of the Zeiss IM-35 microscope.

The laser optical trap was calibrated by viscous drag through the aqueous medium in the microscope focal plane, using a latex bead. The viscous force was generated by oscillatory motion of the bead by a piezoceramic-driven stage (Wye Creek Instruments, Frederick, MD) at a constant velocity. The position of the bead in the trap was tracked using a nanometer-level tracking program (Gelles et al., 1988) to analyze video records of the experiments. Positional variation of the particle in the trap with $60 \mathrm{~mW}$ of laser power entering the microscope was $11( \pm 1.7) \mathrm{nm}$. The calibration showed a linear force-distance relationship for the optical tweezers. To study the variation in trap calibration with height above the coverslip surface, latex beads $(0.5 \mu \mathrm{m}$ diameter $)$ were trapped with the same laser power at different perpendicular positions. There was an increase in particle displacement at $2 \mu \mathrm{m}$ or less from the glass surface that implied a viscous coupling to the coverslip surface. From $2-5 \mu \mathrm{m}$ above the surface, the force on the beads in the trap was constant (Dai and Sheetz, 1995a). This calibration was used to calculate the forces that form tethers. All of these experiments were performed 3-4 $\mu \mathrm{m}$ above the coverslip surface to minimize viscous coupling to the glass surface, and the laser power was monitored simultaneously.

To measure the membrane static tether force $F_{0}$, a rat IgG-coated bead was placed on the cell surface; once the bead bound to the cell it was pulled out at a constant velocity and held for several seconds at a constant tether length $(\sim 15 \mu \mathrm{m})$. In the off-line analysis, the position of the bead in the trap during the stationary phase was measured using the nanometer-scale tracking program (Gelles et al., 1988), and the force of the tether on the bead was calculated from the calibration of the laser trap (Dai and Sheetz, 1995a). To measure the time course of the tether force during cell swelling and shrinking, a tether was formed and then kept at a constant length as osmotic perturbations were effected through the flow cell. When isotonic medium was refreshed by wicking through 


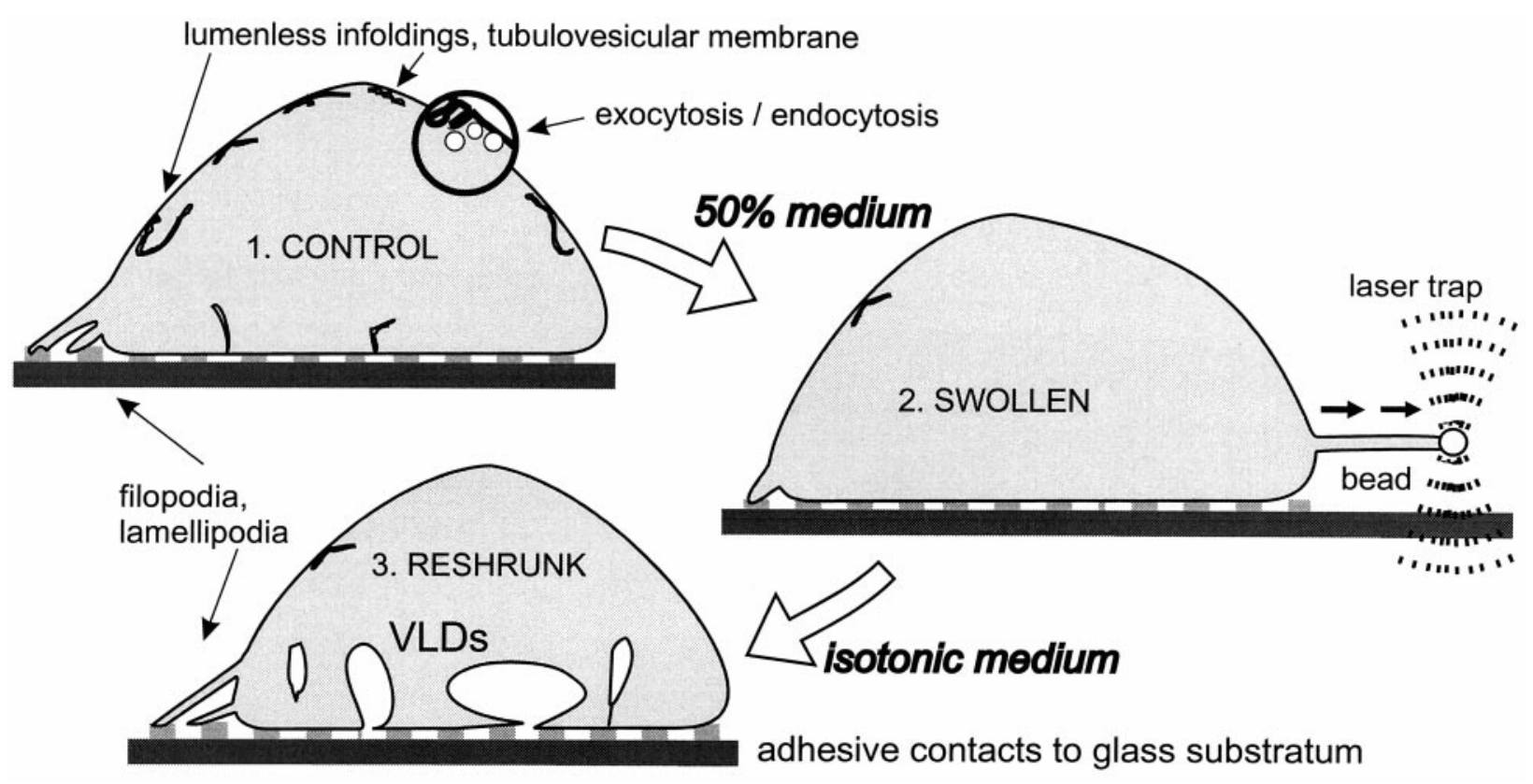

Figure 2. A schematic showing how swelling and then reshrinking, the protocol used here, elicits vacuole-like dilations (VLDs) as invaginations from the substratum. If VLD-bearing neurons are left in isotonic medium, the VLDs disappear by a cell-mediated process termed "recovery" (see Fig. 5). If neurons are made to reswell, VLDs disappear by "reversal" as if VLD bilayer material is pulled back to the general plasma membrane surface (Reuzeau et al., 1995). Membrane tension changes may depend on redisposition of membrane associated with the sources and sinks depicted [lumenless infoldings and/or tubulovesicular membrane, exocytotic and endocytotic membrane, filopodial membrane, VLD membrane; from their high-voltage electron microscopy, Fejtl et al. (1995) suggest that neuronal somata have "hotspots" of mechanically recruitable internal membrane stores]. Tethers were pulled parallel to the substratum, as shown.

the two-coverslip chamber, no persistent tether force changes occurred, indicating that flow per se was not an issue.

Where indicated, neurons were loaded with the membraneimpermeant fast $\mathrm{Ca}^{2+}$ chelator BAPTA (1,2-bis(2-aminophenoxy)ethane- $N, N, N^{\prime}, N^{\prime}$-tetra-acetic acid) by incubating them in $30 \mu \mathrm{M}$ BAPTA-AM (Molecular Probes, Eugene, OR) in 0.3\% DMSO for $1 \mathrm{hr}$. The ester (i.e., the AM form) freely crosses the lipid bilayer; in the cytoplasm, esterases cleave the ester, trapping BAPTA.

Lymnaea neurons were voltage-clamped as described previously (Morris and Horn, 1991; Wan et al., 1995) using the permeabilized-patch configuration to optimize cytoplasmic integrity. To ensure that all data were from cells with no previous osmotic challenge, data were obtained from only one neuron per dish. Only unarborized neurons were used. Osmotic changes were made with electrolytes held fixed; for these experiments, isotonic medium had half the $\mathrm{NaCl}$ of normal saline removed, but osmolarity was restored with sucrose. The medium was made hypotonic $(0.5 \times$ normal $)$ by removing the sucrose or made hypertonic $(1.75 \times$ normal) with additional sucrose. Unarborized neurons $\leq 40 \mu \mathrm{m}$ diameter were used. Neurons were well clamped, as indicated by voltagedependent current responses (fast inward followed by slower outward currents) to depolarizing steps, which were checked from time to time. Capacitance was measured every $10 \mathrm{sec}$ as $Q / V$ (charge/V); $Q$ was obtained by integrating the capacitative current elicited by a $10 \mathrm{mV}$ step. Leak current was excluded. During each $10 \mathrm{sec}$ interval, series resistance compensation was adjusted as necessary. To change the bath medium as quickly as possible without losing the seal, a faster than normal flow was used for $30 \mathrm{sec}$. Data collection was interrupted during the changeover.

\section{RESULTS}

\section{Choice of swelling solution}

In earlier experiments on Lymnaea neurons (Reuzeau et al., 1995), we elicited VLDs with a brief (2-4 min) stepwise solution exchange from isotonic medium to near-distilled water and then back. Here, a less severe hypotonic shock (to $50 \%$ isosmotic for 4 min and then back) was used; because the flow chamber produced very abrupt solution exchanges, easily detectable swelling and then VLDs resulted in all neurons. This protocol therefore was used (1) to measure tether force during VLD formation in the flow chambers, (2) to measure the corresponding changes during VLD formation in mammalian neurons (in which case a $50 \%$ mammalian medium was used), and to (3) to allow for comparison of tether force changes with other changes in Lymnaea neurons [we have previously monitored capacitance and area changes as well as channel activity in a $47 \%$ medium (Wan et al., 1995; Morris and Horn, 1991) and have followed neuronal volume in 30 and $60 \%$ media (Morris et al., 1989)]. Although dilute, $50 \%$ media are not inimical to neuronal function and in fact stimulate neurite outgrowth over the time frame of the experiments described here (Bray et al., 1991; Lin et al., 1995).

\section{Bead binding and tether pulling for tension measurements}

Rat IgG-coated latex beads bound less readily on molluscan neurons than on mammalian (Dai and Sheetz, 1995a) neurons. Approximately $10-20 \%$ of attempts to bind trapped beads to the cell surface were successful but approximately half of the bound beads did not diffuse in the plane of the membrane and so could not be used to pull out tethers with the laser tweezers. Presumably, the immobile beads were anchored to cytoskeleton-linked surface molecules. No morphology changes were observed when the rat IgG-coated bead bound to cell surface.

\section{Static membrane tether forces}

Figure $3(a-d)$ shows video-enhanced DIC micrographs of a tether-pulling sequence for a Lymnaea neuron in isotonic medium. The various steps are illustrated, including binding of a trapped bead to the cell surface $(a)$, pulling the tether at a constant rate with the bead in the laser trap $(b)$, holding the tether (c) at a fixed length for the $\sim 30 \mathrm{sec}$ required to obtain a timeaveraged static tension measurement, and finally, $(d)$ the almost 

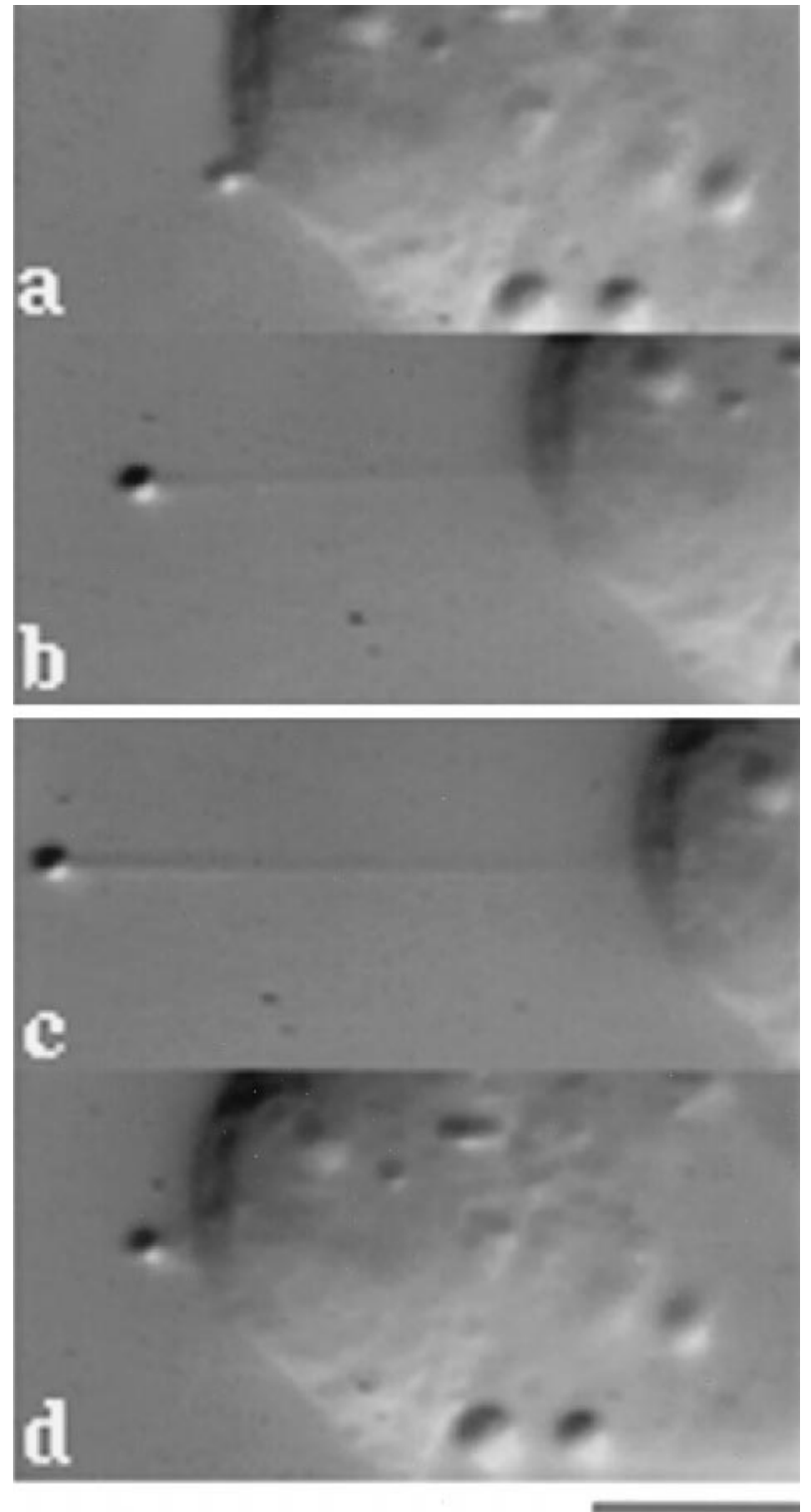

Figure 3. DIC images of a tether-pulling sequence with a Lymnaea neuron. $a$, A bead was held on the cell surface by the laser trap. $b, c$, The tether was formed at a constant pulling velocity. $d$, The complete retraction of the tether when the laser trap was turned off indicates that the static tether was under tension. Scale bar, $4 \mu \mathrm{m}$.

completed retraction of the tether just after the tweezers were turned off. How tether force data are used to extract static tether force measurements is shown in Figure 4 for a neuron in isotonic medium (control), in 50\% medium (swollen), and then back in isotonic medium (reshrunk). Note that the letters $a, b$, and $c$ in Figures 3 and 4 correspond to the same stages. As observed previously, the reshrinking of neurons in isotonic medium was accompanied by the formation of VLDs (Fig. 5). Figure 6, which shows rat hippocampal neurons forming transient VLDs after a brief swelling episode in $50 \%$ medium, illustrates that this phenomenon is not a molluscan specialization. Molluscan neurons were used, however, because ancillary work on the consequences of osmotic perturbations has been performed in them (Herring et al., 1998).

Osmotic perturbations from isotonic (control) to $50 \%$ and then

\section{Measuring Static Tether Force}

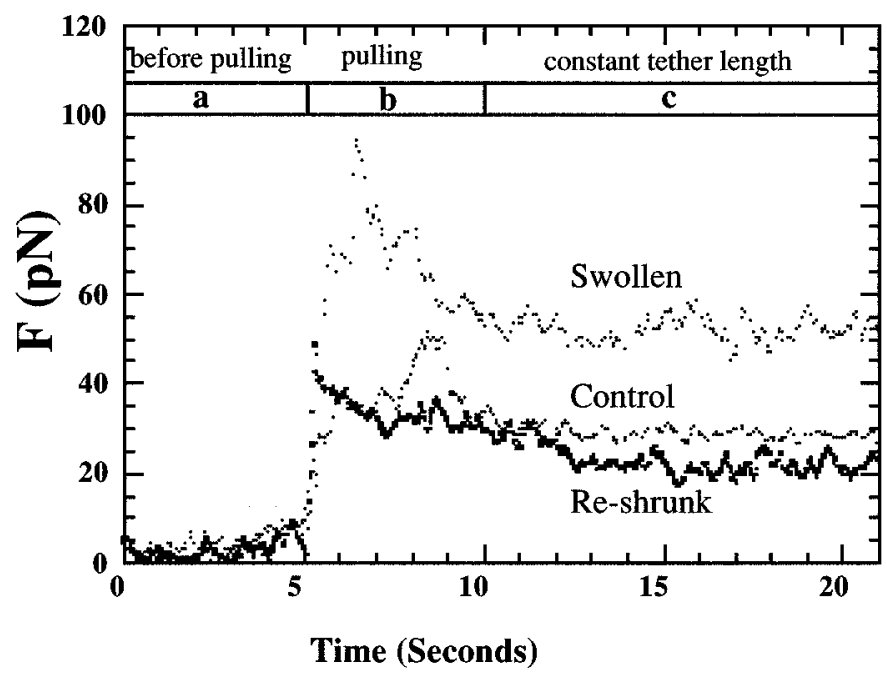

Figure 4. Examples of tether forces during tether formation (an initial transient occurs as tether is pulled to a fixed length) from a neuron under control, swollen, and reshrunk conditions. Static tether force values were obtained from the average tether force when the tethers were held at a constant length. The traces for this neuron illustrate the higher static tether forces typical of swollen neurons.

back to isotonic (reshrunk) medium altered static tether force values. The static force in control neurons was $27.9 \pm 2.1 \mathrm{pN}$ (SEM, $n=30$ ). In neurons swollen in $50 \%$ medium it was $49.5 \pm$ $3.2 \mathrm{pN}(n=32)$, and in reshrunk neurons it was $21.3 \pm 1.7 \mathrm{pN}$ $(n=32)$. The reshrunk value is slightly and significantly $(p<$ 0.02 ) lower than that of control, and the value of the static tether force for swollen neurons is significantly greater than both $(p<$ 0.001).

From the static tether force $\left(F_{0}\right)$ measurements, we can estimate the membrane tension $\left(\mathrm{T}_{\mathrm{m}}\right)$ according to the equation (Sheetz and Dai, 1996):

$$
F_{0}=2 \pi\left(2 B T_{\mathrm{m}}\right)^{1 / 2}
$$

Assuming that the membrane bending stiffness $(B)$ is constant for all experimental conditions and that its value is $2.7 \times 10^{-19} \mathrm{~N} \cdot \mathrm{m}$ [the value estimated for dorsal root ganglion neurons (Hochmuth et al., 1996)], then we can estimate the membrane tensions for the three conditions: for control cells, $\sim 0.037 \mathrm{mN} / \mathrm{m}$; for swollen cells, $\sim 0.12 \mathrm{mN} / \mathrm{m}$; and for reshrunk cells, $\sim 0.021 \mathrm{mN} / \mathrm{m}$. Although the steady-state membrane tension increased approximately threefold with swelling (from 0.04 to $0.12 \mathrm{mN} / \mathrm{m}$ ), this increase falls far short of membrane lytic tensions, which are approximately two orders of magnitude higher $(\sim 10 \mathrm{mN} / \mathrm{m})$. The estimated membrane tension of the swollen neurons is also considerably less than the membrane tension required to activate mechanosensitive channels in most patch recordings (2-10 $\mathrm{mN} / \mathrm{m}$ ) (Gustin et al., 1988; Sachs and Morris, 1998).

We assume that membrane bending stiffness stays constant because it is determined primarily by membrane composition, which should be little affected by osmotic perturbations. If swelling could induce decreases in bending stiffness by expanding membrane, according to Equation 1, this would augment the estimates of tension. However, membrane cannot be expanded more than $3 \%$. 

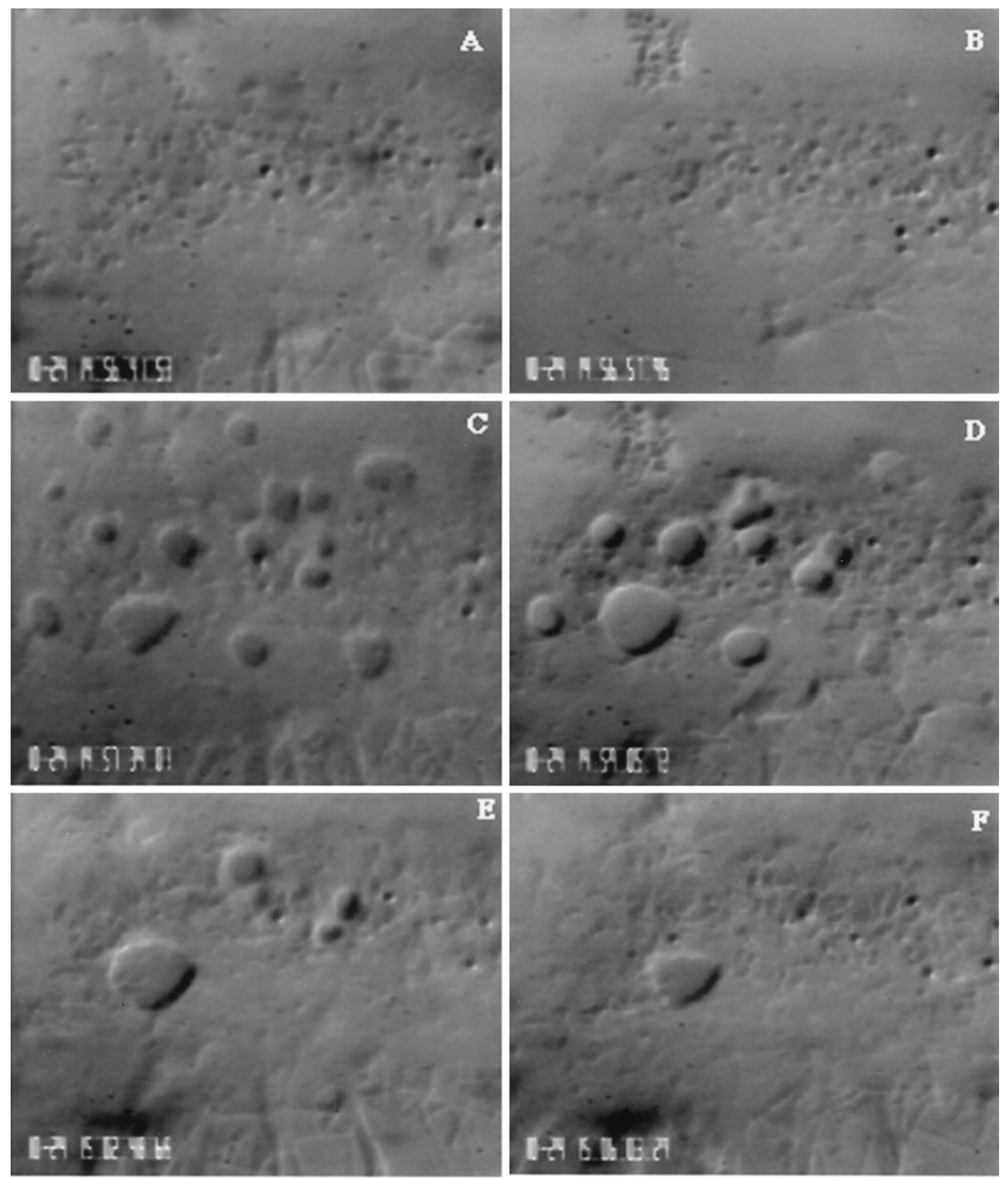

Figure 5. DIC images of a Lymnaea neuronal lamella in a swollen $(A, 50 \%$ medium) and a reshrunk $(B-F$, normal medium) condition, illustrating the VLDs that were always elicited by reshrinking. During 10 min after return to normal medium, the VLDs form $(B, C)$ and then undergo recovery $(D-F)$ VLDs were always evident within 1 min of return to normal medium, as shown, but their recovery was often slower. VLDs also form under the soma but are most easily visualized in lamellar regions. Scale bar, $2 \mu \mathrm{m}$. 

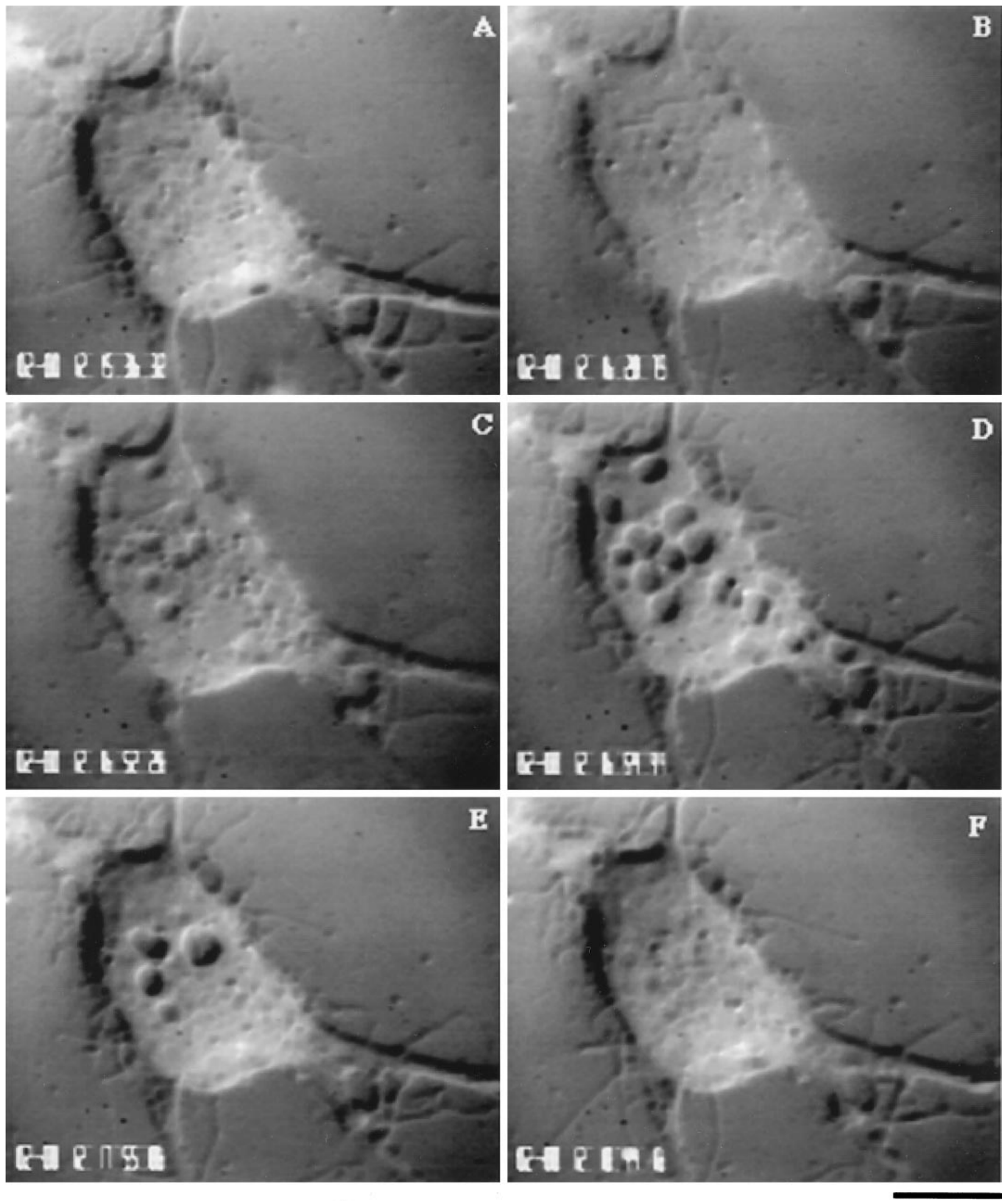

Figure 6. DIC images of an embryonic day 18 rat hippocampal neuron forming VLDs and undergoing recovery at $37^{\circ} \mathrm{C}$. The entire sequence occurred during a period of $<3 \mathrm{~min}$. The neuron was in isotonic medium in $A, C-F$; in $B$, it was swollen in $50 \%$ medium. VLDs formed within seconds of the transition from $50 \%$ to normal medium $(C)$; by $F$, cell-mediated recovery was complete, and the VLDs had disappeared. A subsequent swell-shrink cycle elicited VLDs at the same locations (data not shown), consistent with the repeatability of VLDs at discrete sites seen in cells as diverse as Lymnaea neurons (Reuzeau et al., 1995) and frog muscles (Krolenko et al., 1995). Scale bar, $6 \mu \mathrm{m}$. 

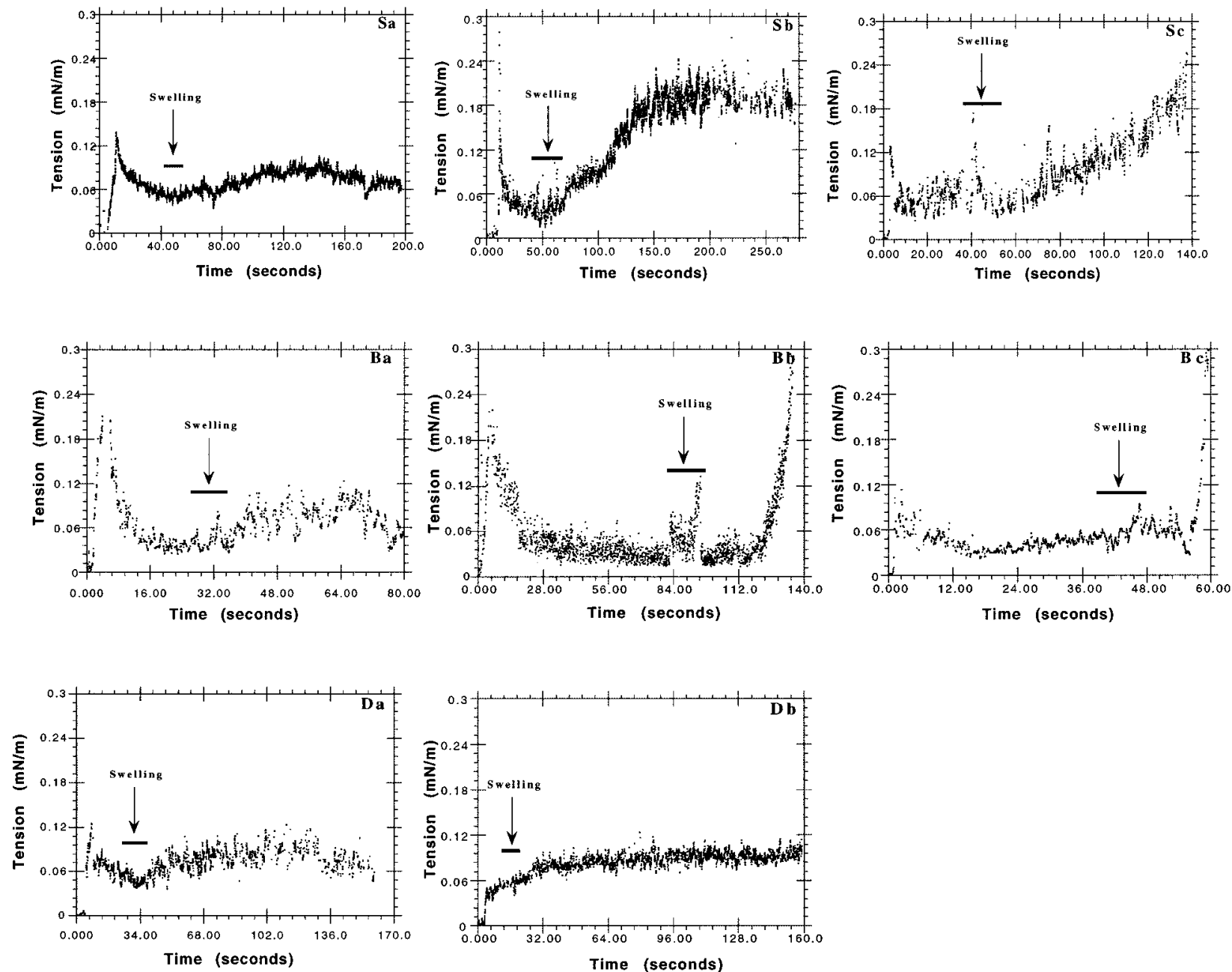

Figure 7. Examples of the time courses for dynamic tether force measurements for control neurons ( $S a, S b, S c)$, for neurons treated in DMSO ( $D a, D b)$ (control for BAPTA-loading), and for BAPTA-loaded neurons $(B a, B b, B c)$. Bars marked swelling represent the time during which isotonic solution was exchanged for $50 \%$ by wicking through the chamber. This procedure per se was no more disruptive with anisotonic than with isotonic exchanges. The data summarized in Table 1 are from such time courses.

\section{Time course of tether forces during swelling and shrinking: dynamics of the response}

Time course tension measurements were made by first forming a tether and then swelling in $50 \%$ medium under one of three conditions: control, pretreatment with DMSO, and pretreatment with BAPTA-AM plus DMSO. We showed previously (Herring et al., 1998) that the BAPTA-loading conditions are effective for Lymnaea neurons, lowering resting cytoplasmic $\left[\mathrm{Ca}^{2+}\right]$ from 100 $\mathrm{nM}$ to $\sim 30-40 \mathrm{nM}$. Moreover, when subjected to a 5 min swelling episode in $50 \%$ medium, BAPTA-loaded neurons show no elevation of cytoplasmic $\left[\mathrm{Ca}^{2+}\right]$ and, in a manner indistinguishable from controls, form VLDs on return to isotonic medium.

A selection of tension time courses for swelling under the three conditions is illustrated in Figure 7; the entire data set is summarized in Table 1. Before swelling, steady-state tensions in BAPTA-loaded cells and in both control conditions (untreated and DMSO-treated) were identical (Table 1), but less than $1 \mathrm{~min}$ after swelling began, most BAPTA-loaded neurons experienced sudden large membrane tension increases whose full extent we could not measure because the force exerted on the bead by the plasma membrane exceeded the trap limit, causing the tethered beads to escape. In controls, this was a rare occurrence. It should
Table 1. Dynamic membrane tension measurements during swelling

\begin{tabular}{|c|c|c|c|c|c|}
\hline \multirow[b]{2}{*}{ Treatment } & \multirow[b]{2}{*}{$n$} & \multirow[b]{2}{*}{$\begin{array}{l}\text { Preswell ten- } \\
\text { sion }(\mathrm{mN} / \mathrm{m})\end{array}$} & \multicolumn{3}{|c|}{$\%$ of neurons showing } \\
\hline & & & $\begin{array}{l}\text { Stable } \\
\text { tension } \\
\text { increases }\end{array}$ & $\begin{array}{l}\text { Transient } \\
\text { tension } \\
\text { increases }\end{array}$ & $\begin{array}{l}\text { Tension } \\
\text { surges }\end{array}$ \\
\hline Control & 24 & $0.043 \pm 0.002$ & 30 & 61 & 9 \\
\hline \multicolumn{6}{|l|}{ BAPTA (in } \\
\hline $0.3 \%$ DMSO) & 12 & $0.042 \pm 0.003$ & 0 & 33 & 67 \\
\hline DMSO $(0.3 \%)$ & 5 & $0.043 \pm 0.004$ & 20 & 80 & 0 \\
\hline
\end{tabular}

be emphasized that although the sudden tension excursions in the BAPTA-loaded neurons were beyond our measurement limit, these abrupt increases did not go on to become cell-rupturing tensions. Whether the measured excursions were irreversible or just the upswing of a larger transient is unknown. The pulled tether is the measurement probe, and membrane tensions large enough to cause bead escape terminate the tension measurement. Nevertheless, it is important to put the present result together with the established finding that BAPTA-loaded Lymnaea neu- 
Figure 8. Time course of the tether force when a Lymnaea neuron was subjected to swell-shrink cycles as indicated. Each time normal medium was exchanged for $50 \%$ medium (swell), tension rose and then fell spontaneously toward a lower steady-state level. Once the spontaneous tension fall was complete (as determined by monitoring a real-time indicator of the laser trap force), the solution was switched back to normal medium (shrink).

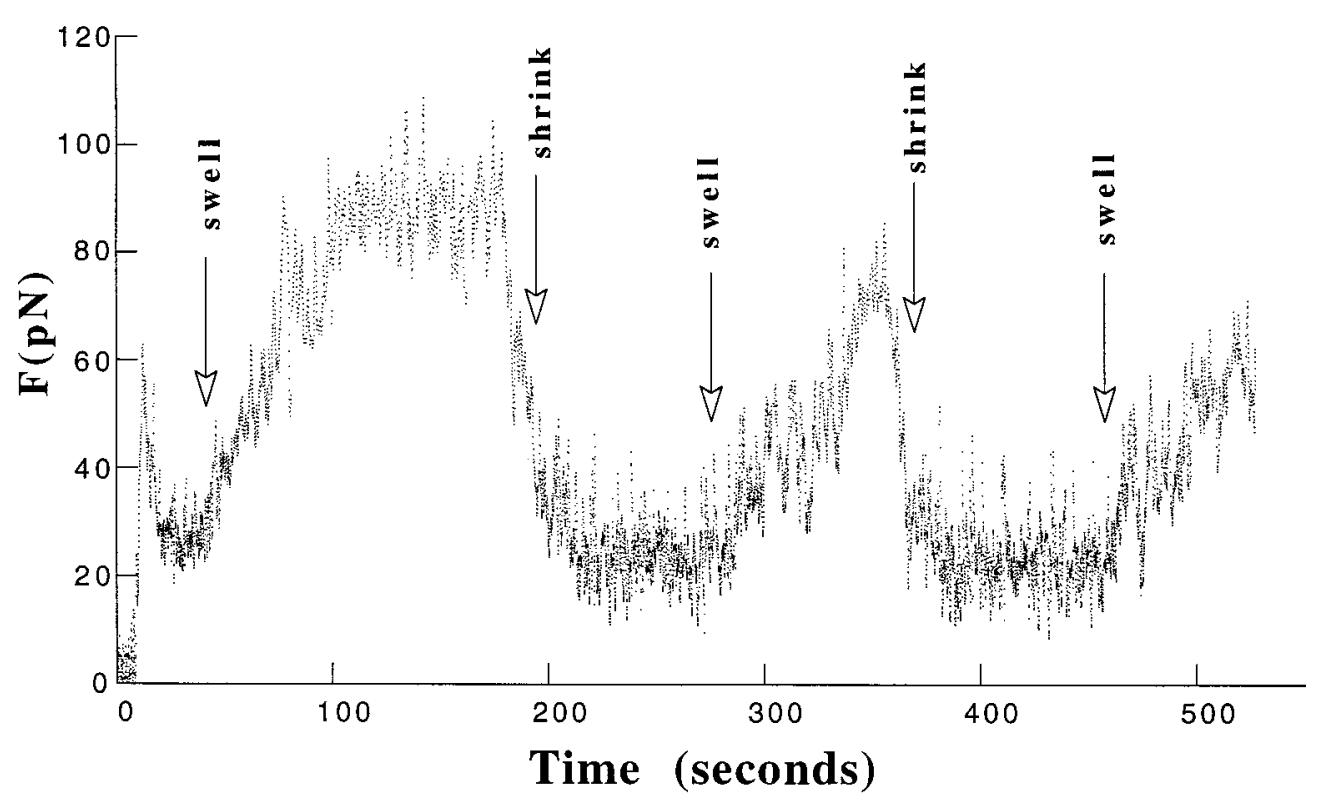

bending stiffness (which depends principally on the molecular composition of the bilayer) during cell swelling and shrinking was relatively constant.

Uncommonly striking tension transients occurred during each swelling episode in Figure 8 (none of the transients noted for control neurons in Table 1 was as dramatic, although surges sometimes occurred). It may be that this time course represents the extreme (in terms of duration and amplitude) of oscillatory events that, by virtue of well functioning feedback, are essentially damped out in most neurons. This would be in keeping with the observation of large tension surges with BAPTA, if the surge is regarded as the upswing of a poorly damped oscillation.

\section{Membrane capacitance changes}

If membrane tension changes trigger membrane area changes, tension and capacitance changes should occur on a similar time scale, with capacitance lagging briefly. Large, long-lasting tension transients like those in Figure 8 would be particularly suitable for generating discernible capacitance increases, but as just mentioned, they were the exception. Step changes of bath tonicity for voltage-clamped Lymnaea neurons proved to be not feasible. For proper space clamp, round unarborized neurons were required, but flow pipes (which can generate step solution changes) brought close to such neurons mechanically disrupted the gigaohm seals, generating unacceptable current noise. Nevertheless, after the bath solution was changed as abruptly as possible, the capacitance of swelling neurons was monitored over a time period similar to that for the dynamic tension measurements.

Figure $10 A, B$ illustrates time courses for neurons just after switching over to hypotonic medium; samples of the capacitative transients used to estimate membrane capacitance are shown. All neurons swelled and increased their capacitance in the 50\% solution. In each of 12 neurons monitored, most (as in $A$ ) or all (as in $B$ ) of the detected capacitance change had already occurred during the first $30 \mathrm{sec}$ after the switch from isotonic to hypotonic. The increase of $8 \pm 1 \%(n=12)$ within 10 min was osmolarityinduced and not, say, a consequence of the $30 \mathrm{sec}$ period of faster solution flow, because if solution was changed to hypertonic, using the same increased flow for $30 \mathrm{sec}$ [either directly from isotonic

saline to hypertonic (data not shown) or from hypotonic to the tether force: the higher the tension, the smaller the tether thickness. This may also suggest that variations in membrane 

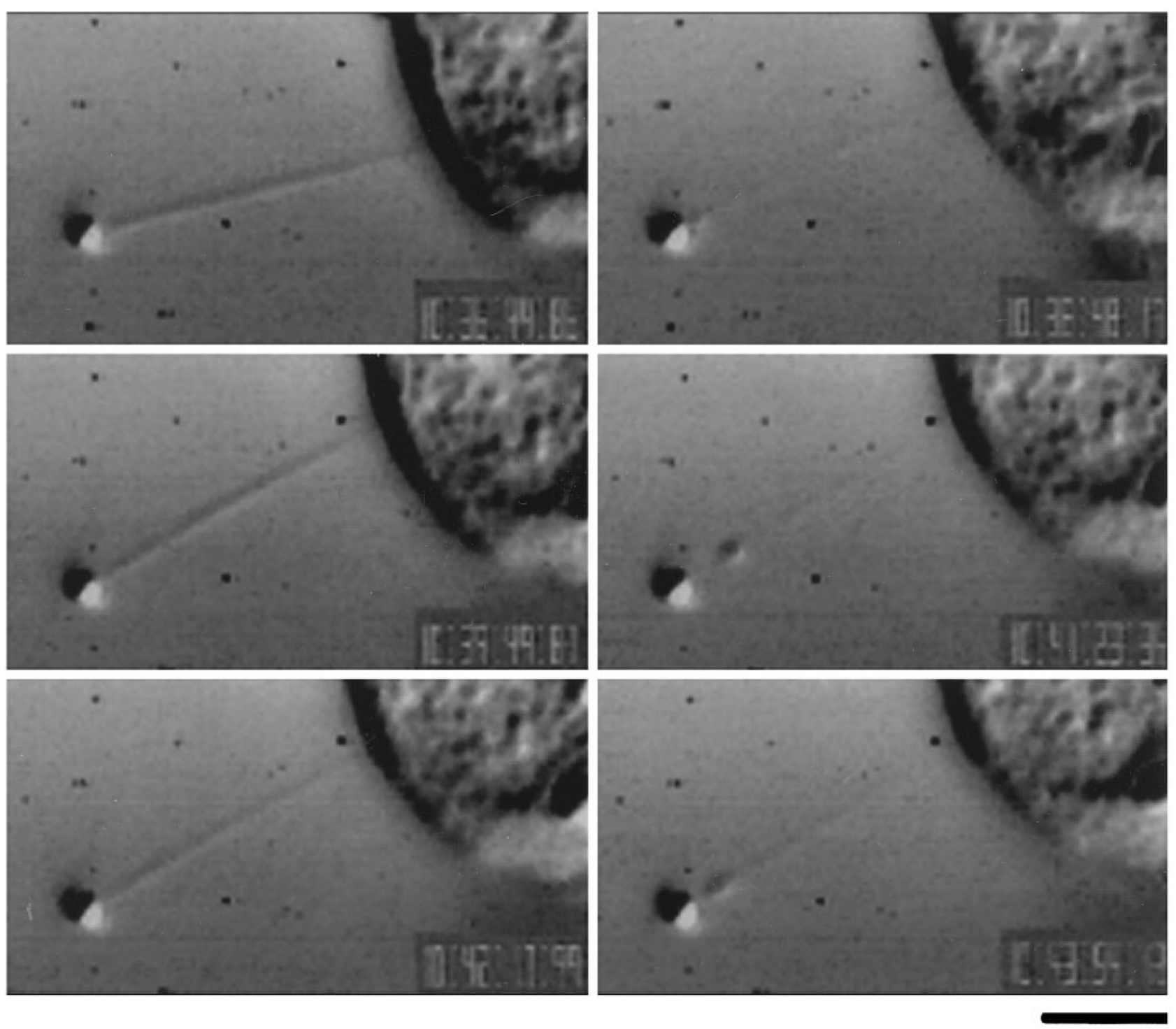

Figure 9. DIC images of a tether pulled from a Lymnaea neuron. The medium is switched repeatedly between normal (left panels) and 50\% (right panels) medium. Note that the tether thickness is less when the neuron is swollen in $50 \%$ medium and that it reverts to its previous size each time normal medium is restored. Scale bar, $2.5 \mu \mathrm{m}$.

hypertonic, as illustrated in Fig. 10B], capacitance invariably decreased. Demonstrating that voltage-clamp quality was good, Figure $10 C$ shows voltage-dependent ionic currents. The neuron was clamped first in isotonic and then in hypotonic medium. Because the extracellular $\left[\mathrm{Na}^{+}\right]$was half normal in both media, the inward followed by outward ionic currents were as expected. The capacitance time courses confirmed that membrane area rapidly increased in swollen neurons.

\section{DISCUSSION}

\section{Overview}

In contrast to epithelial cells, for which large osmotic fluctuations are routine, neurons have no pressing need for osmotic resilience, nor are neurons mechanically active in the manner of muscles or fibroblasts. Yet to an astonishing degree, neurons are osmotically and mechanically resilient. Those used here can swell fivefold without rupture and then recover on return to normal medium (Wan et al., 1995). Vertebrate neurons, too, are nonfragile. In culture they show vigorous outgrowth after a switch to $50 \%$ osmotic medium (Bray et al., 1991). Neurons probably need mechanically robust membranes to cope with the exigencies of extension and remodeling during embryogenesis, organogenesis, neuronal repair, and neuronal plasticity (Morris et al., 1997; Van Essen, 1997). Surprisingly little is known about the basis for membrane resilience. To what extent does it bespeak a tolerance of high membrane tensions and to what extent an ability to prevent large tension excursions?

Because molluscan neurons are large (20- to 100- $\mu$ m-diameter somata) and readily manipulated (patch-clamped, injected, cultured with minimal media and substrata at room temperature), they have proved useful for posing fundamental questions about membrane mechanics and surface area regulation (for references, see Herring et al., 1998). As is evident from Figure 6, however, mammalian neurons exhibit the VLD phenomena (shrinkinginduced invagination of membrane at discrete sites at the substratum, followed by recovery), which initially alerted us to the 
A

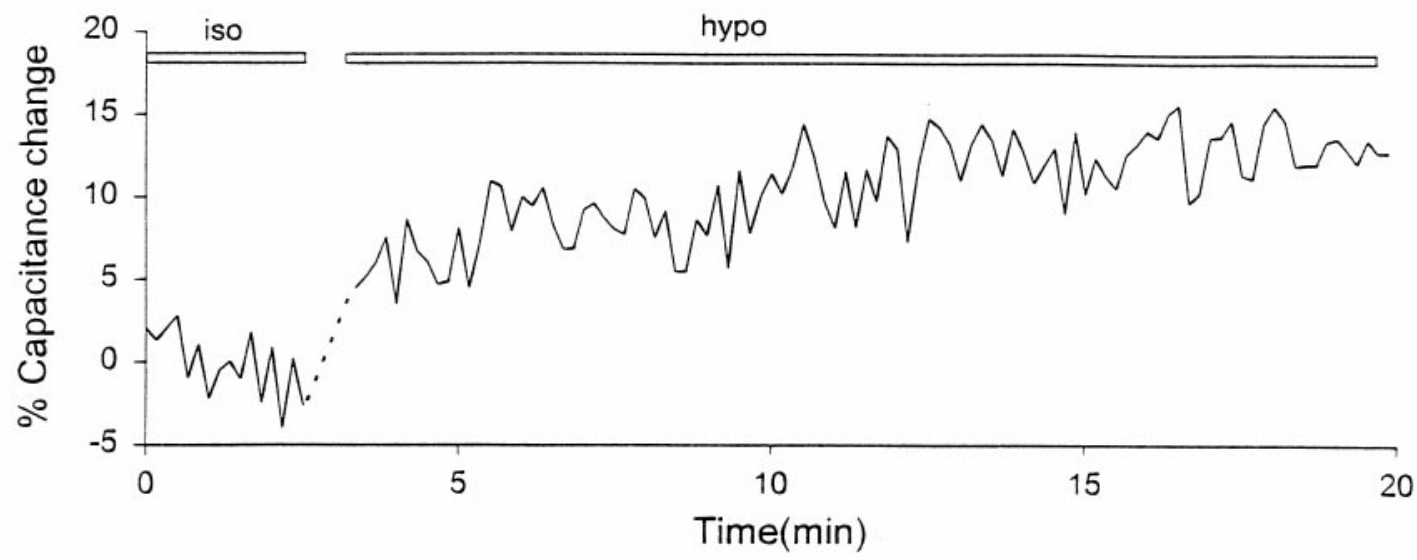

B

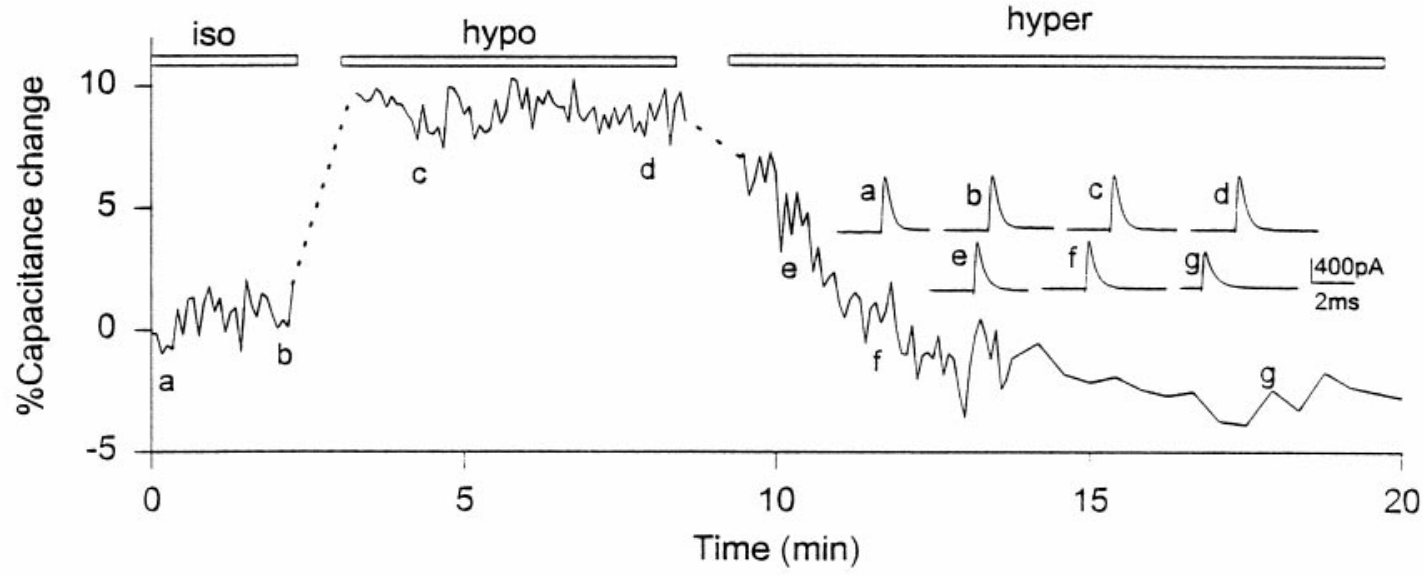

C
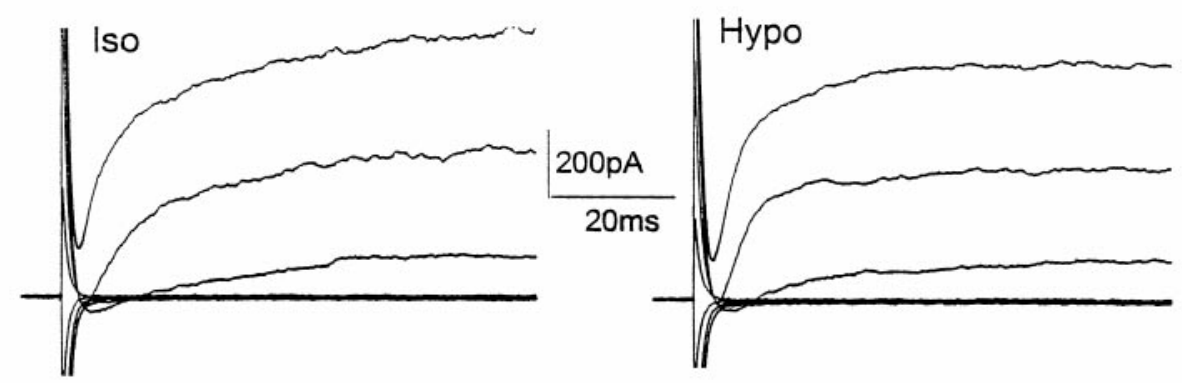

Figure 10. Time courses of membrane capacitance in voltage-clamped Lymnaea neurons. A, Example in which capacitance continued to increase for several minutes after the solution switch. $B$, Example in which it was complete by the time the solution switch finished. In this case, capacitance was also monitored during subsequent shrinkage. Selected capacitance current traces are given from the indicated times. $C$, As explained in the text, good space clamp in the voltage-clamped neurons was confirmed by eliciting ionic currents before and during swelling. iso, hypo, and hyper refer to the tonicity of bathing solutions.

idea that osmotic perturbations elicit surface area regulation (Reuzeau et al., 1995), and so do neuronally differentiated PC12 cells and hippocampal glia among other cells (L. Mills and C. E. Morris, unpublished observation).

As mechanical stimuli, we used swell and then shrink perturbations that first inflated neurons and then, with shrinkage, elicited VLDs.

\section{Swelling}

Swelling consistently yielded small membrane tension increases and reshrinkage produced tension decreases to below control levels. Membrane tension was calculated from the force required to hold a drawn-out membrane tether. For static measurements, tethers were pulled once the osmotic perturbation was completed, whereas for dynamic measurements, tethers were pulled before perturbations. Overall, both static and dynamic tether force data (i.e., for swollen and swelling neurons, respectively) indicated that neurons avoided rather than tolerated high membrane tension. The laser tweezers can hold beads against forces up to $100 \mathrm{pN}$, which would have corresponded to membrane tensions in the range of $0.50 \mathrm{mN} / \mathrm{m}$, yet static tether forces for swollen neurons corresponded to only $0.12 \mathrm{mN} / \mathrm{m}$. 
Swelling neurons loaded with BAPTA, however, did experience surges of tension exceeding the trap limit (Fig. 7, Table 1). Fura-2 measurements of Lymnaea neurons (Herring et al., 1998) showed that in both isotonic and 50\% media, BAPTA lowers cytoplasmic $\left[\mathrm{Ca}^{2+}\right]$ from $\sim 100 \mathrm{nM}$ to $\sim 35 \mathrm{nM}$. BAPTA-loaded neurons do not rupture with repeated swelling in $50 \%$ medium and, like controls, produce VLDs when reshrinking (Herring et al., 1998).

Tension transients occurred in one-third of swelling neurons (Table 1). Tension might increase and then fall by triggering membrane addition. An additional possibility is that cortical contractility could mitigate rising tension (Mills et al., 1994; Glogauer et al., 1998). We found (Wan et al., 1995) that one-third of neurons under extreme osmotic stress writhe slowly as if the cortex were contractile. Writhing is blocked and survival time shortened by $N$-ethylmaleimide, an inhibitor of actomyosin.

A priori, cytoplasmic dilution and impaired $\mathrm{Ca}^{2+}$ buffering each might interfere with tension regulatory mechanisms, so it is not surprising that swelling plus BAPTA was more compromising than swelling alone or BAPTA alone: $<10 \%$ of control neurons versus $>60 \%$ for BAPTA-AM treated neurons experienced tension surges in excess of the trap limit. Tension surges with BAPTA are more reasonably attributed to impaired $\mathrm{Ca}^{2+}$ dependent contractility than failed membrane recruitment (Herring et al., 1998) because swelling neurons gain capacitance independent of $\mathrm{Ca}^{2+}$ [neither $0.5 \mu \mathrm{M}$ bath $\mathrm{Ca}^{2+}$ nor cytoplasmic EGTA prevents swelling-induced capacitance increases (Wan et al., 1995)]. These tension surges, moreover, indicate something more rudimentary; namely that tensions would become treacherously high in $50 \%$ medium were it not for tension-counteracting mechanisms including membrane addition and actomyosin contractility. If the cortical cytoskeleton effects dynamic strengthening of membrane by myosin-based contractility (Wan et al., 1995; Kuwayama et al., 1996), then tension-driven ( $\mathrm{Ca}^{2+}$-independent) membrane addition may occur when hydrostatic forces exceed the combined (passive and active, i.e., structural and contractile) strength of the plasma membrane.

The picture that emerges is as follows. Although not fragile, neuronal membrane relies on compliance and not strength as an anti-rupture strategy in the face of excessive hydrostatic stress. Only a threefold steady-state tension increase was registered with $50 \%$ medium, although (everything being equal) a 100 -fold increase (from $\sim 0.04$ at rest to $\sim 4 \mathrm{mN} / \mathrm{m}$ at rupture) could have been tolerated had there been no compliance in the system. (Rupture tensions for neuronal membranes are discussed below under stretch-sensitive channels.) The ability to avoid high membrane tension is evidently not dependent on subtle biochemical signals, which could be compromised by cytoplasmic dilution.

\section{Reshrinking}

Membrane tension in reshrunk neurons (swollen 4-5 min and then returned to isotonic medium) fell significantly below preswelling levels. Did excess membrane acquired during swelling persist, becoming flaccid with shrinkage? This seems too facile to explain persistent low tension, given that VLD membrane invaginated as neurons shrank. Because swelling stimulates a $\mathrm{K}^{+}$ current in Lymnaea neurons (Wan et al., 1995), solute loss during swelling may contribute to a lower than original volume when the tonicity is restored. Additionally, tension may have gone low because bilayer-cytoskeleton interactions that contribute to resting tension were diluted by the added membrane during swelling, and despite VLD invagination, full retrieval of that membrane may be protracted. During reshrinkage, actin filaments immediately coat VLDs and begin organizing as substratum-adherent leading edge (filopodia and lamellipodia) at the base of VLDs (Cohan et al., 1997). Therefore, reduced membrane tension at the nonadherent surface (where tethers are pulled) may reflect reallocation of cortical F-actin to VLD membrane. F-actin from the dorsal cortex that relocates will be unavailable to exert a membrane osmotic pressure (Fig. 3) on the dorsal bilayer, so tethers pulled there will yield subnormal tensions. Shrinkage evidently creates points of particularly high tension at the substratum (the sites of VLD invagination). The reason for this is not understood, but because VLDs in muscle cells have a spectrin membrane skeleton (Herring, 1998), a bulk flow of bilayer may occur from low-tension dorsal regions to substratum points where membrane skeleton is drawn inward by shrinking cytoplasm.

\section{Tension and neuronal stretch-sensitive channels}

In various experimental systems, membrane rupture occurs at tensions in the 2-12 $\mathrm{mN} / \mathrm{m}$ range (Nichol and Hutter, 1996), and Lymnaea neuron membrane is not exceptional. Aspirated at the tip of patch pipette, it ruptures at a pressure of $\sim 20 \mathrm{kN} / \mathrm{m}^{2}$ (i.e., $-150 \mathrm{mmHg}$ ). Assuming the membrane curvature of a $5-\mu \mathrm{m}-$ diameter sphere and using Laplace's Law [tension $=$ (pressure $\times$ diameter)/4], this corresponds to rupture at $\sim 5 \mathrm{mN} / \mathrm{m}$. Under whole-cell clamp, rupture occurs at $\sim 1 \mathrm{kN} / \mathrm{m}^{2}$ (Morris and Horn, 1991; Wan et al., 1995), which in a $20 \mu \mathrm{m}$ neuron would generate a tension of $12 \mathrm{mN} / \mathrm{m}$. Thus, Lymnaea neuron membrane ruptures at $5-12 \mathrm{mN} / \mathrm{m}$, and the expected range is $2-12 \mathrm{mN} / \mathrm{m}$.

Mechanosensitive channels, including those of Lymnaea (Morris and Horn, 1991) and Aplysia (Vandorpe and Morris, 1992) neurons, generally exhibit mechanosensitive gating only at tensions that are near lytic. Because we found that $50 \%$ medium, an egregiously large hypotonic stimulus in physiological terms, generates only a sustained threefold increase in membrane tension, sustained stretch-activation of channels is unlikely. When swelling augments $\mathrm{K}^{+}$currents (Wan et al., 1995), it is likely that increased membrane area or second messengers are responsible.

\section{Membrane dynamics in neurons and tension regulation}

In many cells, including growing neurons, there is a rapid exchange of membrane between the plasma membrane and internal stores. On axons, membrane could be added at $\sim 50 \mathrm{~mm}^{2} / \mathrm{min}$ at the growth cone and taken in at the cell body (Dai and Sheetz, 1995b). Under swelling conditions, endocytosis is inhibited in mast cells (Dai et al., 1997). For a typical neuron, the surface area of $\sim 2000 \mathrm{~mm}^{2}$ would be increased by $8 \%\left(160 \mathrm{~mm}^{2}\right)$ in $3 \mathrm{~min}$ at that rate. Alternatively, the tubulovesicular membranes of the axon, a putative source of membrane, could be drawn out at a rapid pace.

We have proposed that as swelling elevates membrane tension, the mechanical energy serves to recruit membrane, lowering the plasma membrane tension by increasing its area. The large abrupt perturbations used here may demand that neurons recruit membrane from emergency stores, whereas more gradual variations in membrane tension may trigger recruitment from other sources. Where appreciable amounts of membrane are constitutively exocytosed and endocytosed, stalling endocytosis via elevated tension may suffice to increase membrane area and ease tension. With different rates of swelling and different levels of constitutive membrane trafficking, different mechanisms may participate to different extents. The intermediary steps between tension and increased membrane area are not understood, but in principle, 
direct tension-induced recruitment would seem to be more failsafe than recruitment regulated by tension-modulated chemical signals. For dynamic cells to regulate their surface membrane area and volume, there must be a complex relationship between membrane trafficking, cytoskeleton, and solute fluxes. Cell shape and volume interplay to define a given state of surface area, and arguably, membrane tension is a parameter that the cell could use dynamically and locally to determine the proper balance. How tension changes are transduced into volume and surface area changes is not known. Several-fold tension increases may not suffice to activate volume-sensitive currents but perhaps may alter interactions between regulatory subunits and ion channels. Finally, we caution that our results refer to measurements accessible by pulled tethers. During osmotic perturbations, membrane adjacent to cell-cell or cell-substratum adhesions may experience tensions exceeding those that we observed.

\section{REFERENCES}

Bray D, Money NP, Harold FM, Bamburg JR (1991) Responses of growth cones to changes in osmolality of the surrounding medium. J Cell Sci 98:507-515.

Cohan CS, Welnhofer EA, Herring TL, Morris CE (1997) Actin and filopodial dynamics in osmotically perturbed molluscan neurons. Mol Biol Cell 8:256a.

Czekay R, Kinne-Saffran E, Kinne RKH (1994) Membrane traffic and sorbitol release during osmo- and volume regulation in isolated rat renal inner medullary collecting duct cells. Eur J Cell Biol 63:20-31.

Dai J, Sheetz MP (1995a) Mechanical properties of neuronal growth cone membranes studied by tether formation with laser optical tweezers. Biophys J 68:988-996.

Dai J, Sheetz MP (1995b) Axon membrane flows from the growth cone to the cell body. Cell 83:693-701.

Dai J, Sheetz MP (1995c) Regulation of endocytosis, exocytosis and shape by membrane tension. In: Protein kinesis: dynamics of protein trafficking and stability, pp 571-576. New York: Cold Spring Harbor Laboratory Press.

Dai J, Ting-Beall HP, Sheetz MP (1997) The secretion-coupled endocytosis correlates with membrane tension changes in RBL $2 \mathrm{H} 3$ cells. J Gen Physiol 110:1-10.

Evans EA, Yeung A (1994) Hidden dynamics in rapid changes of bilayer shape. Chem Phys Lipids 73:39-56.

Fejtl M, Szarowski DH, Decker D, Buttle K, Carpenter DO, Turner JN (1995) Three-dimensional imaging and electrophysiology of live Aplysia neurons during volume perturbation: confocal light and high-voltage electron microscopy. JMSA 1:75-85.

Fink RD, Cooper MS (1996) Apical membrane turnover is accelerated near cell-cell contacts in an embryonic epithelium. Dev Biol 174:180-189.

Fujimoto Y, Ogawa K (1989) Retrieving vesicles in secretion-induced rat chromaffin cells contain fodrin. J Histochem Cytochem 37:1589-1599.

Gelles J, Schnapp BJ, Sheetz MP (1988) Tracking kinesin-driven movements with nanometer-scale precision. Nature 331:450-453.

Glogauer M, Arora P, Chou D, Janmey PA, Downey GP, McCulloch CAG (1998) The role of actin-binding protein 280 in integrindependent mechanoprotection. J Biol Chem 273:1689-1698.

Goslin K, Banker G (1992) Rat hippocampal neurons in low density culture. In: Culturing nerve cells (Banker G, Goslin K, eds), pp 252281. Cambridge, MA: MIT.

Gustin MC, Zhou X-L, Martinac B, Kung C (1988) A mechanosensitive ion channel in the yeast plasma membrane. Science 242:762-765.
Heidemann SR, Buxbaum RE (1994) Mechanical tension as a regulator of axonal development. Neurotoxicology 15:95-108.

Herring T (1998) Calcium, actin and the spectrin skeleton: responses during swelling and shrinking perturbations. MSc thesis, University of Ottawa.

Herring T, Baltz JM, Morris CE (1998) Mechanosensitive membrane disposition: can tension directly recruit new membrane to the surface in swelling neurons? Mol Biol Cell 7:450a.

Hochmuth RM, Shao J, Dai J, Sheetz MP (1996) Deformation and flow of membrane into tethers extracted from neuronal growth cones. Biophys J 70:358-369.

Krolenko SA, Amos WB, Lucy JA (1995) Reversible vacuolation of the transverse tubules of frog skeletal muscle: a confocal fluorescence microscope study. J Muscle Res Cell Motil 16:401-411.

Kuwayama H, Ecke M, Gerisch G, Van Haastert PJ (1996) Protection against osmotic stress by cGMP-mediated myosin phosphorylation. Science 271:207-209.

Lin C, Lamoureux P, Buxbaum RE, Heidemann SR (1995) Osmotic dilution stimulates axonal outgrowth by making axons more sensitive to tension. J Biomech 2812:1429-1438.

Mills JW, Schwiebert EM, Stanton BA (1994) Evidence for the role of actin filaments in regulating cell swelling. J Exp Zool 268:111-120.

Morris CE, Horn R (1991) Failure to elicit neuronal macroscopic mechanosensitive currents anticipated by single channel studies. Science 251:1246-1249.

Morris CE, Williams B, Sigurdson WJ (1989) Osmotically-induced volume changes in isolated cells of a pond snail. Comp Biochem Physiol 92A:497-483.

Morris CE, Lesiuk H, Mills LR (1997) How do neuronal processes monitor their mechanical status? Biol Bull 192:118-120.

Nichol JA, Hutter OF (1996) Tensile strength and dilatational elasticity of giant sarcolemmal vesicles shed from rabbit muscle. J Physiol (Lond) 493:187-198.

Pybus J, Tregear RT (1975) The relationship of adenosine triphosphatase activity to tension and power output of insect flight muscle. J Physiol (Lond) 247:71-89.

Reuzeau C, Mills LR, Harris JA, Morris CE (1995) Discrete and reversible vacuole-like dilations induced by osmo-mechanical perturbations of neurons. J Membr Biol 145:33-47.

Sachs F, Morris CE (1998) Mechanosensitive ion channels in nonspecialized cells. Rev Physiol Biochem Pharmacol 132:1-78.

Sheetz MP, Dai J (1996) Modulation of membrane dynamics and cell motility by membrane tension. Trends Cell Biol 6:85-89.

Smith PG, Tokui T, Ikebe M (1995) Mechanical strain increases contractile enzyme activity in cultured airway smooth muscle cells. Am J Physiol 268:L999-1005.

Sukhorukov VL, Arnold WM, Zimmermann U (1993) Hypotonically induced changes in the plasma membrane of cultured mammalian cells. J Membr Biol 132:27-40.

Vandorpe DH, Morris CE (1992) Stretch-activation of the Aplysia S-channel. J Membr Biol 127:204-214.

Van Essen DC (1997) A tension-based theory of morphogenesis and compact wiring in the central nervous system. Nature 385:313-318.

Wan X, Harris JA, Morris CE (1995) Responses of neurons to extreme osmotic stress. J Membr Biol 145:21-31.

Wartenberg M, Hamann J, Pratsch I, Donath E (1992) Osmotically induced fluid-phase uptake of fluorescent markers by protoplasts of Chenopodium-album. Protoplasma 166:61-66.

Waugh RE, Song J, Svetina S, Zeks B (1992) Local and nonlocal curvature elasticity in bilayer membrane by tether formation from lecithin vesicles. Biophys J 61:974-982. 